\title{
STAATSANGEHÖRIGKEIT, NATIONALITÄT UND RELIGION IN ISRAEL ${ }^{\cdot 1}$
}

\author{
Von Yitzhak Goldfine und Avner H. Shaki
}

\section{Die Problematik}

Die Frage „Wer ist Jude?“ wurde Ende der fünziger Jahre wieder aktuell. Damals verlangten Nichtjüdinnen, die Juden geheiratet hatten und israelische Bürger geworden waren, daß sie und ihre Kinder aus der Mischehe in ihren Ausweispapieren als Angehörige der jüdischen Nation eingetragen werden sollten ${ }^{2}$. Bei der Frage nach der Religionszugehörigkeit bestand nie ein Zweifel daran, daß diese Frauen und Kinder keine Juden waren. Der Streit entzündete sich an dem Verlangen, zum Zwecke der Eintragung in den Ausweispapieren in Israel auch weltlich als Juden anerkannt zu werden und insoweit die entgegenstehenden Religionsgesetze außer acht zu lassen, also die Frage nach der Eigenschaft als Jude unterschiedlich zu beantworten: für alle die Person, ihren Status und ihre Familienverhältnisse betreffenden Angelegenheiten nach den Religionsgesetzen, zum Zwecke der Registrierung hingegen nach den weltlichen Gesetzen. Im letzteren Fall sollten die religiösen Kriterien hinter den weltlichen zurücktreten.

Der Streit ist bis heute aktuell geblieben und erreichte vor kurzem seinen Höhepunkt in einem Rechtsstreit des israelischen Bürgers Biniamin Schalit gegen den Innenminister vor dem obersten Gericht Israels. Die Entscheidung des Gerichts veranlaßte die Vorlage eines neuen Gesetzentwurfes, nach dem für die Anerkennung als "Jude" nur die Religionsgesetze maßgebend sein sollen. Die vorliegehende Abhandlung will zu den hieran in der öffentlichen Diskussion bekanntgewordenen religiösen, rechtlichen und ideologischen Aspekten kritisch Stellung nehmen.

\section{Die Fragen Ben Gurions}

Bekannt und häufig zitiert ist die Sammlung von Fragen des früheren israelischen Premierministers Ben Gurion an 45 jüdische Gelehrte. Ben Gurion, der selbst gegen die Zugrundelegung religiöser Kriterien bei der Frage nach der Eigenschaft als Jude ist, wandte sich u. a. mit folgenden Fragen an viele Gelehrte in der ganzen Welt: Welchen rechtlichen Status besitzen die Kinder aus Mischehen, bei denen nur der Vater Jude und die Mutter Nichtjüdin ist? Kann dieser Status nur nach den Religionsgesetzen bestimmt werden oder genügt für eine Regi-

\footnotetext{
* Das Verhältnis von Staat und Religion ist eine Kernfrage moderner Staatlichkeit. Goldfine und Shaki behandelten sie im vorliegenden Beitrag an Hand der Frage, ob Nationalität in Israel religiös oder weltlich verstanden werden soll. Von einem ganz anderen Blickwinkel aus taucht sie bei Rahn, unten S. 335 auf, der sich mit der Problematik einer religiösen Partei beschäftigt. Auch in dem Beitrag von Wehner, oben S. 285, ist dem Verhältnis von Kirche und Staat ausführliche Beachtung geschenkt. Die Red.

1 In der Arbeit werden folgende Abkürzungen verwendet:

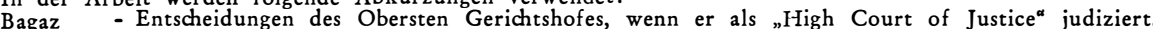
Hapraklit - Der Name der in Tel-Aviv von der Anwaltskammer herausgegebenen rechtswissenschaftlichen

P. D. - "Piskei Din “ - (Entscheidungen) - amtliche Sammlung der Entscheidungen des Obersten Gerichtshofes $1948-1970$.

2 In Israel unterscheidet man für Registrierungszwecke unter israelischen Staatsbürgern nach Nationalität (Jude, Araber, Druse) und Religion (Jude, Mohammedaner, Christ). .
} 
strierung der Kinder als Juden, daß ihr Vater Jude ist? In den Fragen wurde besonders hervorgehoben, daß Israel ein Staat mit vielen Einwanderern aus Ländern sei, in denen die Mischehe fast üblich sei, und daß ein Festhalten an den religiösen Kriterien für viele ein Hindernis zur Einwanderung darstellen würde. Die Antworten auf die Fragen Ben Gurions waren selbstverständlich nicht einheitlich. Doch überwog bei 37 der 45 Befragten, darunter Rabbiner aus allen bekannten Kirchen in den USA, die Auffassung, daß zur Feststellung der Eigenschaft als Jude auch in Zukunft an den religiösen Kriterien festzuhalten sei. Denn dies sei die einzige Möglichkeit, das jüdische Volk vor einer Überfremdung zu bewahren.

Die Feststellung als Jude nach religiösen Kriterien geschieht im wesentlichen unter zwei Gesichtspunkten:

1. Ob jemand von einer jüdischen Mutter geboren ist (Babli³, III., 10, s. 2) oder

2. ob jemand, der nicht von einer jüdischen Mutter geboren ist, nach den Religionsgesetzen konvertiert hat.

Der erste Anknüpfungspunkt geht zurück auf ein Wort der Bibel (5. Buch Mose, 7.3): „Du sollst sie (die nichtjüdischen Frauen) nicht heiraten“, da das von einer Nichtjüdin geborene Kind auch nicht Jude ist.

\section{Die Bedeutung der Registrierung}

Die meisten Probleme, die sich um den Begriff "Jude" ranken, beziehen sich auf die Registrierung der Nationalität und Religion in den Einwohnermeldeämtern sowie auf das Einwanderungsgesetz, wie z. B. in dem später behandelten Fall „Rufeisen“.

Die Registrierung in den Einwohnermeldeämtern verlief eine Zeitlang ohne Probleme, bis immer häufiger verlangt wurde, daß in den Rubriken „Religion“ und "Nationalität" entweder "religionslos" oder "Jude“, auch wenn die Mutter Nichtjüdin war, oder "ohne Nationalität" registriert werden. Im Lande wuchs die Unsicherheit, worauf dann Ben Gurion seine berühmten Fragen an die jüdischen Gelehrten richtete.

Aus dieser Zeit stammen zwei Anordnungen des Innenministeriums: eine des Ministers Bar-Jehuda, der Mitglied der sozialistischen Partei „Leachdut Haawoda“ war und dessen Anordnungen zur Frage der Registrierung liberal weltlich waren; die andere Anordnung stammt von dem Mitglied der religiösen Partei, Minister H. M. Schapira, und hat bis heute Gültigkeit.

Der inzwischen verstorbene Innenminister Bar Jehuda bestimmte in seiner bis zum Jahre 1960 geltenden Anordnung,, daß der Registrierungsbeamte im Einwohnermeldeamt in gutem Glauben an die Erklärung des Anmeldenden, dieser sei z. B. "Jude" nach seiner Religion und Nationalität oder "Jude" nach seiner Nationalität und "religionslos" nach seinem Glauben, die entsprechende Eintragung ohne Nachprüfung nach religiösen Kriterien vornehmen sollte.

Der spätere Innenminister M. H. Schapira hat „Verwaltungsanordnungen für den Registrierungsbeamten "5 erlassen, in denen er festlegte, daß als Jude in der

3 Babilonische Talmud

4 Takanot Haswut-1956 (Anordnungen Sammlung Nr. 609 vom 31. 5. 1956, S. 820).

5 Hanchaiot Sar Hapnim, H. M. Schapira von 1960 (Kwiat Miwchan Halacha). 
Rubrik „Religion“ oder „Nationalität" nur eingetragen werden dürfe, wer

a) von einer jüdischen Mutter geboren ist und keine andere Religion hat, oder

b) wer zum Judentum übergetreten ist.

Mit dem Hervorheben einer anderen Religionszugehörigkeit wollte man betonen, daß zum Christentum konvertierte Juden, die zwar als Juden geboren waren, nicht als Juden registriert werden können. Auch diese Einschränkung hat viel Kritik in der Ơffentlichkeit hervorgerufen.

\section{Die rechtliche Bedeutung der Anerkennung als „Jude“}

Die Hauptbedeutung der religiösen Zugehörigkeit einer Person im israelischen Recht liegt im Bereich des Familien- und Erbrechts. Die beiden wichtigsten Gebiete: Ehe und Scheidung unterstehen ausschließlich religiösen Gerichten. Für Juden besitzen die Rabbinatsgerichte die ausschließliche Gerichtsbarkeit gemäß dem Gesetz über die Rabbinatsgerichte von 19536. Hiernach sind nicht nur die Gerichte mit religiösen Richtern besetzt, sondern auch das anwendbare materielle Recht ist religiöses Recht ( $\$ 2$ des Gesetzes). Andere Angelegenheiten des Familien- und Erbrechts, wie Unterhalt für Frau und Kinder, Vormundschaft, Testamente usw. werden sowohl von religiösen wie auch weltlichen Gerichten behandelt, wobei das materielle Recht wiederum mit wenigen Ausnahmen das religiöse Recht ist. Außerdem taucht der Begriff "Jude" in manchen Gesetzen sowie in der feierlichen Deklaration über die Entstehung des unabhängigen Staates Israel (Unabhängigkeits-Deklaration) auf. Aus diesen Gesetzen soll kurz zitiert werden:

a) „Unabhängigkeits-Deklaration“ (Veröffentlicht: Offiziell Gazette Nr. 1 vom 14. 5. 48): „In Eretz, Israel, entstand das jüdische Volk ... im Jahre 1897 hat der zionistische Kongreß getagt auf Anregung neuzeitlichen „Propheten“, Theodor Herzel, (der die Gründung eines jüdischen Staates 50 Jahre zuvor vorausgesagt hat), um das Recht des jüdischen Volkes auf Begründung eines eigenen Nationalstaates in seiner alten Heimat zu proklamieren. Dieses Recht wurde in der Balfour-Deklaration anerkannt und wurde vom Völkerbund im Mandat bestätigt, welches ausdrücklich die historische Verbundenheit zwischen dem jüdischen Volk und dem Land Israel als neuem nationalen Heim für die Juden betonte. Am 29. November 1947 veröffentlichte das Komitee der Vereinten Nationen eine Resolution über die Gründung eines jüdischen Staates Israel in Eretz ISRAEL. Wir deklarieren hiermit die Entstehung eines jüdischen Staates in Eretz, Israel, den Staat Israel“.

b) In der Verordnung über die Verwaltung und Recht 19487. Paragraph 18a bestimmt über den Sabbat und die Feiertage in Israel: Die beiden Neujahrstage, der Kippur Tag, der erste und achte Tag Sukot, der erste und siebte Tag Pesah und die Schawuotfeier sind die gesetzlichen Feiertage im Staate Israel. Nichtjuden haben das Recht, ihre Feiertage nach ihrer Ordnung einzuhalten, d. h. der Sabbat und die israelischen Feiertage sind gesetzliche Feiertage nur für Juden.

6 Chok Sipput Batei Din Rabaniim (Nisuin we-Gerusin) - 1953 (Gesetzbuch 1953/165).

7 Pkudat Sidrei Hasilton we-Hamispat - 1948

(Offizielle Gazette Nr. 2 vom 21. 5. 1948, S. 1). 
c) In dem Gesetz über die Arbeits- und Ruhestunden von $1951^{8}$ wurden die Arbeits- und Ruhetage für Arbeiter in Israel festgelegt, und es wurden wiederum ausdrücklich die Feiertage für Juden und Nichtjuden festgelegt.

d) In den israelischen Gesetzen sind zwei wichtige Anordnungen zu finden:

(1) Art. $1^{9}$ :

Jeder Jude hat das Recht, nach Israel zu kommen . . .

(2) Art. $2^{10}$ :

Ein Jude, der gemäß dem Einwanderungsgesetz einwandert, erhält automatisch die israelische Staatsangehörigkeit.

In diesem Gesetz kam die Tendenz der zionistischen Bewegung zum Ausdruck, jedem Juden, gleichgültig woher er kommt, die Einwanderung in seine Heimat zu ermöglichen.

Nach dieser Auffassung ist jeder Jude in der Welt ein potentieller Bürger des StaatesIsrael (s. o. die Zitate von der Unabhängigkeitserklärung, vgl. GG \166 [1]. Es gibt noch weitere Gesetze, in denen der Begriff "Jude“ auftaucht, wie z.B. das Gesetz über die Registrierung von Eheschließungen und Scheidungen von 1919 oder das Familienrechtsergänzungsgesetz über Alimente von 1959 und andere, die aber nicht wesentlich für die vorliegende Abhandlung sind.

Erwähnenswert ist nur noch, daß in dem Gesetz über die Einwohnermeldung von 1965'11, das Streitgegenstand bei der Registrierung der Nationalität und Religion war, das Wort "Jude“ überhaupt nicht erscheint.

\section{"Jude“ in den Quellen und der Gesetzgebung}

Bevor der Verfasser mit einer Analyse der großen Entscheidungen in Israel über die Frage „Wer ist Jude?" beginnt, soll hier ein Versuch zur Aufklärung des Begriffs "Jude" unternommen werden.

Das Wort "Jude" hat seine eigene Geschichte. Heute und seit relativ langer Zeit bedeutet dieses Wort eine bekannte tägliche Bezeichnung für Angehörige des jüdischen Volkes in Israel und außerhalb. In früheren bis hinab zu den biblischen Zeiten war der Begriff „Bnei-Israel“ (Kinder-Israel), oder "Israel“ oder "Israelit“ der verbreitetste. Auch später während des Mittelalters wurden diese Bezeichnungen beibehalten. Ursprünglich bezeichnete man als "Juden" die Bewohner des Landes "Judä“. Aber nachdem es kein israelisches Königreich mehr gab, umfaßte der Begriff "Jude“ alle Angehörigen des Staates Israel, auch außerhalb des Landes. In Jeremia $(40,12)$ steht: „Und alle Juden sind zurückgekehrt zum Land Juda von allen Plätzen, wo sie hingeschoben wurden“; und dann in (36,21): „Und der König sandte den Juden die Rolle abzuholen" (s. auch Könige II 16,25, Zacharias 8,33, Buch Ester, Esra 4,12, Nechemias 2,16). In der Welt des antiken Rom wurde der Bewohner des Landes Judea "Judeus" genannt, daneben benutzte man den Begriff „Hebreus“. Auch die Päpste und Bischöfe benutzten den Begriff "Judea" und „Hebreas" (so auch im neuen Testament).

Später, zu Zeiten Ferdinands und Isabellas wurden in Spanien alle Nicht-Christen als "Juden" bezeichnet. Vom Lateinischen und Griechischen wanderte der Begriff

\footnotetext{
8 Chock Seot Haawoda we-Hamenuha - 1951 (Gesetzbuch 1951/204).

9 Chock Haswut -1950.

10 Chock Haezrachut -1952 (Gesetzbuch Nr. 95 vom 8. 4. 1952, S. 146).

11 Chock Mirsam Uchlusin - 1965 (Gesetzbuch 1965, S. 270).
} 
"Jude" in alle europäischen Sprachen. Erst seit der Zeit der Emanzipation in Europa begann man zwischen den Begriffen "Jude" und "Israelit" zu unterscheiden. In manchen jüdischen Gemeinden benutzte man den Begriff „Israelit“, um die religiös-gläubige im Gegensatz zur national-ethnischen Zugehörigkeit zu bezeichnen. Nur das letztere Begriffspaar bezog sich auf den Begriff "Jude“. Da ist die Zeit der Trennung zwischen Staat und Religion in Ländern wie Frankreich und Deutschland (Israelitische Religionsgemeinde in Deutschland z. B.). Es ist vielleicht erwähnenswert, daß diejenigen, die noch zur zionistischen Bewegung standen, den Begriff "Jude“ beibehielten. Herzel z. B. nannte sein Buch „Der Judenstaat", zwei von den wichtigsten Zeitschriften hießen "Jüdischer Verlag" und "Jüdische Rundschau“, Martin Buber nannte seine Zeitschrift „Der Jude“.

\section{Der Fall Rufeisen ${ }^{12}$}

\section{Der Sachverhalt}

Der Fall betrifft einen Juden, der vor seiner Einwanderung in Israel zum Christentum übergetreten und katholischer Priester geworden war. Er fühlte sich jedoch trotz seiner Konvertierung und der katholischen Priesterschaft als Jude und beantragte, ihn als "Einwanderer" im Sinne des Einwanderungsgesetzes zu behandeln. Im Laufe des Prozesses stellte sich heraus, daß er seinerzeit ein treuer und mit seinem Volk verbundener Jude war und während der Nationalsozialistischen Zeit sogar sein Leben riskiert hatte, um anderen Juden zu helfen.

Die israelische Einwanderungsbehörde war nicht einverstanden, ihn als Einwanderer und damit als "Juden" zu betrachten. Daher beantragt er beim Obersten Gerichtshof, daß es den Innenminister verpflichten solle, ihm gemäß $\ 3$ des Einwanderungsgesetzes von 1950 einen Einwanderungsausweis auszustellen und ihn gemäß $₫ 7$ der Verordnung über die Registrierung der Bevölkerung von 1949 unter der Rubrik „Nationalität“ als Juden einzutragen.

Das Hauptargument von Rufeisen für seine Anerkennung als "Jude“ ist das von ihm behauptete Auseinanderfallen von „jüdischer Religion“ und „jüdischer Nationalität". Rufeisen meinte, er sei zwar nach seinem Glauben ein Christ, nach seiner Nationalität aber Jude, und daß hierin kein Widerspruch liege.

\section{Die Entscheidung des Gerichts}

Das Gericht lehnte den Antrag von Rufeisen mit einer Mehrheit von 4:1 Stimmen ab. Nach Meinung des Gerichts ist der Begriff "Jude“ gemäß dem Einwanderungsgesetz nach weltlichen und nicht nach religiösen Kriterien zu bestimmen. Rufeisen könnte zwar nach dem Grundsatz des Judentums, daß auch ein Jude, der gesündigt hat, Jude bleibt, als Jude angesehen werden. Doch müsse man unter Zugrundelegung weltlicher Kriterien der allgemeinen Überzeugung im Volk Rechnung tragen, wonach ein Jude, der konvertiert hat, sich damit nicht nur außerhalb der jüdischen Religion, sondern auch außerhalb der jüdischen Nationalität

12 Rufeisen gegen den Innenminister, Bagaz 76/62 P. D. XVI. S. 2428. S. auch Badas gegen Sadeh Civil Appeal 174/65, P. D. XX Teil 1, S. 617; Gitia gegen das Hauptrabbinat, Bagaz 359/66, P. D. XXII Teil 1, 290. 
gestellt hat. Die Mehrheit der jüdischen Richter machte somit keinen Unterschied zwischen „jüdischer Religion“ und „jüdischer Nationalität" und bestritt die von Rufeisen behauptete Möglichkeit, daß jemand nach seiner Religion Christ und nach seiner Nationalität Jude sein kann.

\section{Gedanken zur Mehrheitsentscheidung}

Nach Meinung des Oberrichters Silberg kann der Fall nicht auf religiöse Kriterien abgestellt werden. Demgemäß sei Rufeisen, da er von einer jüdischen Mutter geboren wurde, als Jude anzuerkennen, so daß die seine Person betreffenden Angelegenheiten zur Zuständigkeit des Rabbinatsgerichts gehörten.

Diese von Silberg und anderen vertretene Meinung stieß auf Kritik, vor allem des Internationalprivatrechtlers Dr. Shaki. Nach diesem scheint Rufeisen zwar auf den ersten Blick tatsächlich Jude zu sein, da er das Kriterium des religiösen Rechts erfülit, von einer jüdischen Mutter geboren zu sein, doch habe er sich aus folgenden Gründen durch seine freiwillige Konvertierung aus der jüdischen Religion ausgeschlossen:

a) Auf Rufeisen treffe der Grundsatz, daß Jude auch der bleibt, der einmal gesündigt hat, auf Grund seines Übertritts zum Christentum nicht mehr zu. Der Rambam, größter jüdischer Philosoph des Mittelalters, unterscheidet zwischen Juden, die eine einfache Sünde begangen haben, und Juden, die gegen sämtliche Mosaische Gesetze verstoßen haben. Nur für erstere treffe obiger Grundsatz zu. Dagegen sei ein Jude, der zum Christentum übergetreten ist, zweifellos ein „Konvertit für die gesamte ,Tora“ (Fünf Bücher Moses)“, der sich aus dem Judentum selbst ausgestoßen hat und in keiner Beziehung mehr Jude ist.

b) Nach den Lehren der jüdischen Religion erwirbt ein Jude sein Recht auf Heimat durch Erbfolge, ein Konvertit erbe aber nicht nach religiösem Recht.

c) Nach Meinung der religiösen Juden in Israel fallen unter den Begriff "Jude“ im Gesetz über die Rabbinatsgerichte nur solche Juden, die sich voll zum Judentum bekannt haben, wovon aber bei konvertierten Juden keine Rede mehr sein könne.

Ein Vertreter dieser Meinung ist das Mitglied des großen Rabbinatsgerichts, der Rabbiner S. Israeli, der das Gesetz über Ehe und Scheidung nur auf Juden, nicht aber Konvertierte anwenden will. Daher könne ein Konvertit auch ohne weiteres in der Kirche statt vor dem Rabbinatsgericht heiraten, ohne daß jemand auf die Idee käme, diese Ehe etwa wegen Verstoßes gegen das jüdische Ehegesetz $z u$ annullieren. Nur ein Jude, der noch immer zum Judentum gehört, falle unter den Begriff "Jude“ im jüdischen Ehe- und Scheidungsgesetz. Wer sich dagegen durch Konvertierung aus dem Judentum ausgeschlossen habe, unterstehe nicht mehr diesem Gesetz.

Hätte das oberste israelische Gericht diese Definition des Begriffs "Jude" durch das israelische Rabbinat gekannt, so hätte es sich gar nicht erst in eine Diskussion über eine verschiedene Bedeutung des Begriffs "Jude“ — je nach weltlichen und je nach religiösen Kriterien - eingelassen und den Begriff "Jude“ nach einheitlichen Gesichtspunkten definiert. 


\section{Die Meinung des Oberrichters Cohen (Minoritätsentscheidung)}

Der Oberrichter Cohen wollte Rufeisen als Juden i. S. des Einwanderungsgesetzes anerkennen und ihm den Einwandererstatus geben. Für Cohen sind die wesentlichen Kriterien für die Anerkennung als Jude, ob sich der Betreffende als Jude fühlt und sein in gutem Glauben gestellter Antrag auf Registrierung als Jude. Es gebe keinen Widerspruch zwischen der religiösen Zugehörigkeit Rufeisens zur katholischen Kirche und seiner nationalen Zugehörigkeit zur jüdischen Nation. Seine innere Verbundenheit mit dem Staate Israel und dem jüdischen Volk, die in seinem Antrag auf Registrierung als Jude ihren Ausdruck finde, genüge, um Rufeisen als Angehörigen der jüdischen Nation anzuerkennen und ihm alle Rechte eines Juden zu verleihen.

Am konsequentesten führt der Oberrichter Cohen die obengenannte Meinung fort, indem er nur auf die innere Einstellung des Betreffenden, also nur auf subjektive Kriterien abstellt. Im Gegensatz hierzu stehen die objektiven Kriterien des religiösen Rechts: Abstammung von einer jüdischen Mutter oder Übertritt.

Die orthodoxen Juden werden und können das subjektive Kriterium niemals akzeptieren, da sonst $\mathrm{zu}$ viele, die mit dem Judentum nicht das geringste zu tun haben, auf den Gedanken kommen könnten, sich als Juden zu betrachten. Nach ihnen bietet auch der gute Glaube an die Zugehörigkeit zum Judentum keine ausreichende Gewähr gegen einen Mißbrauch.

\section{Eine Meinung aus der Lehre}

Der Jerusalemer Zivilrichter Tedeshi' ${ }^{13}$ interpretiert das Einwanderungsgesetz nach weltlichen und nicht nach religiösen Kriterien. Nach ihm soll es keine Rolle spielen, ob jemand von einer jüdischen Mutter oder einem jüdischen Vater abstammt. In beiden Fällen soll der Betreffende als Volljude angesehen werden. Wenn jemand sich so sehr als Jude fühlt, daß er nach Israel einwandern will, so sollte man ihn auch nach dem Einwanderungsgesetz als Jude betrachten. $\mathrm{Da}$ es sich hier um ein weltliches Gesetz handele, dürfe auch nicht auf religiöse Kriterien abgestellt werden. Doch bei einem zum Christentum übergetretenen Juden vertritt auch Tedeshi grundsätzlich die Meinung, daß sich dieser damit aus der jüdischen Gemeinschaft ausgeschlossen habe. Allerdings hält $\mathrm{T}$. eine andere Beurteilung dann für möglich, wenn der Konvertit in seinem Bewußtsein Jude bleibt und den Willen hat, auch weiterhin Jude zu bleiben. Als Beispiel führt er Benjamin Disraeli an.

Obwohl Tedeshi mit dem Oberrichter Cohen auf subjektive Kriterien abstellt, befürwortet er wegen der besonderen Umstände im Fall Rufeisen die Mehrheitsentscheidung des Gerichts. Da Rufeisen nicht nur einfacher Konvertit, sondern sogar Priester und Mönch ist, der außerhalb der jüdischen Gesellschaft in einem Kloster leben will, habe er nicht die Absicht, sich in die jüdische Gesellschaft einzugliedern. Dies sei aber Voraussetzung für seine Anerkennung als jüdischer Einwanderer im Sinne der zionistischen Bedeutung dieses Begriffes.

13 Hapraklit, XIX, S. 101; vgl. Perles, Hapraklit 1954, September, S. 172; Globus, Hapraklit 1954, September, S. 231. 


\section{Der Fall Schalit ${ }^{14}$}

In diesem erst vor kurzem vor dem Obersten Gerichtshof Israels entschiedenen Prozeß ging es wieder um die Frage „Wer ist Jude?“. Die Entscheidung gehört zu den wichtigsten, die je in Israel gefällt worden sind.

Das Gericht hatte in großer Besetzung mit neun Richtern über den Antrag des Herrn Schalit zu entscheiden, den Innenminister zu veranlassen, die beiden minderjährigen Kinder des Herrn Schalit aus seiner Ehe mit einer Nichtjüdin, die auch nicht zum Judentum übertreten wollte, als Angehörige des jüdischen Volkes zu registrieren. Das Gericht gab mit 5:4 Stimmen Mehrheit dem Antrag statt und wies den Innenminister an, die Kinder von Registerbeamten unter der Rubrik „Nationalität" als Juden eintragen zu lassen. Die Registrierung ist dann auch wenig später erfolgt.

\section{Der Sachverhalt}

Da die Tatsachen dieses Falles typisch sind für viele andere Fälle, sollen sie ausführlich dargestellt werden.

a) Angaben zur Person des Vaters Biniamin Schalit: Er wurde in Israel geboren. Beide Eltern sind Juden. Am 8. 11. 1948 ließ er im Einwohnermeldeamt unter der Rubrik „Religion“ „ohne Religion“ eintragen und die Rubrik „Nationalität" streichen.

In seinem Antrag auf Ausstellung eines Personalausweises erklärte er sich am 7. 12. 1966 als Angehöriger einer „hebräischen Nation“.

Im Jahre 1964 ließ er in der Geburtsurkunde seines Sohnes seine eigene Nationalität als "jüdisch" und im Jahre 1967 in der Geburtsurkunde seiner Tochter als „israelisch“ eintragen. In einem Brief an den Innenminister vom 4. 3. 67 bezeichnete Schalit sich als "Jude nach seiner Erziehung und nationalen Zugehörigkeit".

b) Angaben zur Person der Ehefrau und Mutter der Kinder:

Sie heiratete Herrn Schalit am 29. 12. 1958 in England. Gemäß Herrn Schalits Brief an den Innenminister ist sie in England als Tochter eines schottischen Vaters und einer französischen Mutter geboren.

Am 29. 2. 1960 wurde sie bei Erteilung der Aufenthaltserlaubnis vom Einwohnermeldeamt als "ohne Religion" und mit „britischer Nationalität" registriert. In einem Brief an den Innenminister vom 18. 4. 67 erklärte Herr Schalit, daß seine Frau im Zeitpunkt der Einwanderung in Israel zur „britischen Nation“ gehörte, jetzt aber die „jüdische oder hebräische oder israelische Nationalität“ besitze. Frau Schalit hatte niemals einen Antrag auf Eintragung einer anderen Nationalität gestellt.

In der Geburtsurkunde seines Sohnes bezeichnete Herr Schalit die Nationalität seiner Frau als „britisch“ und in der Geburtsurkunde seiner Tochter als „israelisch".

c) Angaben über die Kinder:

Der Sohn wurde am 14. 3. 64 geboren und nach Angaben des Vaters vom Registrierungsbeamten als Angehöriger der „jüdischen Nationalität“ eingetra-

14 Schalit gg. Innenminister u. a. Bagaz 58/68 (noch nicht offiziell veröffentlicht). 
gen. Diese Eintragung wurde später korrigiert, indem die Eintragung der Nationalität gestrichen und in der Rubrik „Religion“ notiert wurde: „Vater Jude, Mutter Nichtjüdin“.

Die Tochter wurde am 11. 2. 67 geboren. Im Einwohnermeldeamt wurde bei ihr unter der Rubrik „Nationalität“ eingetragen: „Vater Jude, Mutter Nichtjüdin“, während in der Rubrik „Religion“ die Notiz stand: „nicht registriert". Der Vater kannte diese beiden Eintragungen nicht.

In einem Schreiben vom 1. 3. 67 bat Herr Schalit den Registrierungsbeamten, die Nationalität seiner Tochter mit „hebräisch oder jüdisch“ zu bezeichnen. In einem weiteren Schreiben vom 4.3.67 beantragte er, die Nationalität seiner beiden Kinder als "jüdisch" zu registrieren, während er in einem dritten Schreiben vom 21. 5. 67 für beide Kinder wieder verlangt, ihre Nationalität als „jüdisch oder hebräisch“ zu bezeichnen.

\section{Die Urteilsbegründung}

Die Urteilsgründe geben keine Antwort auf die allgemeine Frage: „Wer ist Jude?“, sondern behandeln im wesentlichen die Frage, ob der Registrierungsbeamte bei Anwendung der vom Innenminister nach religiösen Kriterien erlassenen Verwaltungsanweisungen richtig gehandelt hat. Nach Meinung des Gerichts hat das Parlament den Innenminister nienıals zum Erlaß derartiger Anweisungen ermächtigt. Das Gericht kommt daher in seiner Mehrheit zu der Entscheidung, daß die Kinder gemäß der Erklärung ihres Vaters vom Registrierungsbeamten als Juden hätten eingetragen werden müssen.

\section{Kritik}

Die die Entscheidung tragenden fünf Richter betonen, daß sie nicht die Frage entscheiden wollen: „Wer ist Jude?", sondern daß es sich bei dem vorliegenden Fall um rein formell-technische Probleme handele. Die Registrierung im Einwohnermeldeamt geschehe nur aus rein statistischen $Z$ wecken.

Hiergegen ist zweierlei einzuwenden. Einmal leuchtet nicht ein, daß in dem Urteil ein breiter Raum den maßgeblichen Kriterien für die Bestimmung des Begriffs "Jude" gewidmet wird, wenn es sich bei der Frage der Anerkennung als "Jude“ lediglich um ein statistisches Problem handeln soll. Zum anderen geht die Bedeutung der Nationalität für den einzelnen über rein statistische Zwecke hinaus. Zum Beispiel dient ein Personalausweis nicht selten der Information über seinen Inhaber durch Dritte, die sich von den Angaben über die Nationalität häufig in ihren Entscheidungen z. B. über eine Einstellung des Betreffenden beeinflussen lassen. Aber selbst wenn statistische Zwecke im Vordergrund stünden, fragt man sich, welchen Wert überhaupt eine Statistik hat, die nicht der Wahrheit entspricht.

\section{Vergleichende Betrachtung der Fälle „Rufeisen“ und „Schalit“}

Vergleicht man beide Fälle, so erkennt man, daß die Entscheidungen nicht miteinander übereinstimmen. Im Fall Rufeisen lehnte es das Gericht ab, jemanden 
nach seiner Religion als Christen und gleichzeitig nach seiner Nationalität als Juden zu betrachten. Es vermied damit die problematische Unterscheidung zwischen der Religion und der Nationalität eines Juden. Im Fall Schalit hingegen macht das Gericht diesen Unterschied, indem es trotz der Registrierung Herrn Schalits als religionslos seine Kinder als "Juden" betrachtet. Aus der Entscheidung im Fall Schalit folgt, daß es für die Anerkennung der jüdischen Nationalität keine Rolle spielt, welche Religion die betreffende Person besitzt oder ob sie ohne Religion ist, sondern daß jeder gemäß seiner Erklärung als Jude eingetragen werden kann.

\section{Die Meinung der im Fall Schalit überstimmten Richter}

Der Oberrichter Silberg sieht es als unmöglich an, bei einem Juden zwischen Religion und Nationalität zu unterscheiden, und betont den religiös-geistigen Charakter der jüdischen Nationalität. Nach ihm gleicht es einem Verrat am jüdischen Volk, wenn man versucht, die jüdische Nationalität von ihren religiösen Quellen zu trennen.

Der Oberrichter Agranat bezieht seine Entscheidung aus einer Definition des nach seiner Meinung speziellen Begriffs der jüdischen Nationalität. Nach Agranat haben sich die Juden niemals nur als religiöse Sekte betrachtet, sondern als nationale Gemeinschaft, deren typischer Hauptcharakter der Beziehung zu der monotheistischen Religion der Bibel ist. Daraus schließt er die vollkommene Identität zwischen jüdischer Religion und Nationalität und die Unmöglichkeit ihres Auseinanderfallens.

\section{Nationalität und Staatsangehörigkeit in Israel}

In Israel sind die Begriffe Nationalität und Staatsangehörigkeit im Gegensatz zu anderen Staaten nicht identisch, und zwar aus folgenden Gründen

1. Im israelischen Gesetz über das Einwohnermeldewesen werden beide Begriffe nebeneinander gebraucht. Daraus ist zu entnehmen, daß der Gesetzgeber mit dem Begriff „Nationalität" aus ethnischer Sicht bestimmen will, ob jemand "Jude“ oder "Araber" ist, während mit dem Begriff „Staatsangehörigkeit" die Zugehörigkeit zu einem bestimmten Staat (Israel, England usw.) gemeint ist.

2. Wenn in Israel die Begriffe identisch wären, so bedeutete dies, daß sämtliche in Israel lebenden Juden, Araber, Drusen usw. eine Nation bildeten. Dies ist zwar logisch nicht ausgeschlossen, entspricht aber nicht den tatsächlichen Verhältnissen. Die Araber in Israel fühlen sich als Angehörige der arabischen Nation, so wie sich die Juden in der ganzen Welt als Angehörige einer - in der ganzen Welt existierenden jüdischen Nation fühlen.

3. Sollte die jüdische Nationalität mit der israelischen Staatsangehörigkeit identisch sein, so bedeutete das, daß 11 Millionen Juden, die außerhalb Israels leben, nicht zur jüdischen Nation gehören. Eine solche Vorstellung ist unvorstellbar.

Der Teil der israelischen Bevölkerung, der der Mehrheitsentscheidung im Fall Schalit zustimmt und sie unterstützt, ist davon überzeugt, daß in Israel tatsächlich eine jüdische Nation existiert.

Es ist eine jüdisch-israelische Nation, welche mit der jüdischen Religion nichts gemeinsam hat. Eine solche Nationalität, wie z. B. die von Schalit, bedient sich nicht der religiösen Kriterien. Sie charakterisiert eine neue jüdische israelische Nation, 
die in Israel entstand. Wenn man diesen Teil der Bevölkerung in Israel fragt, welche Merkmale diese Nation hat, so lautet die Antwort meist wie folgt:

a) Sie hat ihren Staat, lebt darin und liebt ihn,

b) spricht hebräisch,

c) dient im Militär,

d) hat irgendeine Verbindung zur jüdischen Kultur.

Gegen diese Merkmale einer neuen Nation argumentiert der religiöse Teil der Bevölkerung, daß sie einen Teil der Araber und vor allem die Drusen umfaßt. Die Drusen z. B. sind in Israel geboren, leben dort und lieben ihre Heimat, sie sprechen ausgezeichnet hebräisch, dienen im Militär und haben Beziehungen zur jüdischen Kultur, vor allem zur hebräischen Literatur, israelischen Kunst usw. Jedoch ist es nach diesem Teil der Bevölkerung unmöglich, die Drusen als Juden zu betrachten, und es ist auch noch nie vorgekommen, daß ein Druse sich als Jude bezeichnete. Die Hauptgefahr sieht der religiöse Teil der Bevölkerung darin, daß wenn man sich als eine neuentstandene Nation betrachtet und nicht als eine Fortsetzung der tausendjährigen jüdischen Geschichte, so würde die Argumentation vom historischen Recht dieser Nation auf ihre alte Heimat ihren Sinn verlieren. Dagegen wird oft von dem nicht-religiösen Bevölkerungsteil eingewendet, daß nach religiösen Kriterien der Sohn einer jüdischen Mutter, der mit arabischen Untergrundkämpfern gegen Israel kämpft, als voller Jude betrachtet werden müsse, während das Kind aus einer Mischehe (die Mutter ist Nichtjüdin), das in der israelischen Armee kämpft und bereit ist, für seine Heimat zu sterben, kein Jude wäre. Zur Zeit ist dieses Problem eines der größten innenpolitischen Probleme Israels und wird zweifellos noch lange die israelische Offfentlichkeit, Parlament, Regierung und Gerichte beschäftigen. Einen Hinweis auf die gegenwärtige Tendenz gibt das vor wenigen Tagen vom israelischen Parlament verabschiedete Gesetz zur Verbesserung des Einwanderungsgesetzes von 1950, in dem die religiöse Definition des Begriffs "Jude“ vom Gesetzgeber übernommen wurde. In dem neuen Gesetz heißt es, Jude ist, wer von einer jüdischen Mutter geboren wurde oder wer zum Judentum konvertiert ist. Die religiösen Parteien im Parlament kämpfen für die Definition: „Wer nach dem Thorangesetz zum Judentrum konvertiert. "Damit wollen sie die Anerkennung einer reformistischen Konvertierung vermeiden. Die Reformisten halten sich nicht streng an die religiösen Gesetze und benutzen moderne und bequemere Mittel in ihrer Religionsausübung. Das israelische Parlament kam dem Verlangen der religiösen Parteien nicht nach und entschloß sich zur Anerkennung der reformistischen Konvertierung. Mit diesem Entschluß wurde die schon vorhandene Fülle von Problemen um ein weiteres bereichert. 